\title{
Commission 49: Interplanetary Plasma and Heliosphere
}

\author{
PRESIDENT: David F. Webb \\ VICE-PRESIDENT: Jean-Louis Bougeret \\ ORGANIZING COMMITTEE: Hilary V. Cane, Neil F. Cramer, \\ Stephen W. Kahler, Masayoshi Kojima, Blai Sanahuja, \\ Marek Vandas, Frank Verheest, and Rudolf von Steiger
}

\begin{abstract}
Commission 49 covers research on the solar wind, shocks and particle acceleration, both transient and steady-state, e.g., corotating, structures within the heliosphere, and the termination shock and boundary of the heliosphere. During the last three years there was considerable progress made in studies of solar energetic particles, compositional and other signatures in the heliosphere, solar wind pickup ions, the termination shock, which was finally crossed by a spacecraft, and the boundary between the heliosphere and interstellar medium, and in solar wind modeling and space weather. These topics have been summarized here in five articles, each with extensive references that will guide the reader who wants further details. Observations from the following spacecraft have extensively used during this period: Ulysses, Cassini, Voyager 1 and 2, MESSENGER, ACE, Genesis, SOHO, Wind, and RHESSI.
\end{abstract}

Keywords. interplanetary medium, Sun: solar-terrestrial relations, Sun: coronal mass ejections, Sun: magnetic fields, acceleration of particles

\section{Solar Energetic Particles}

David Lario

(JHU-Applied Physics Lab., Laurel, MD, USA; david.lario@jhuapl.edu)

The fleet of spacecraft distributed over the heliosphere during the maximum phase of the solar cycle 23 has allowed us to improve our knowledge of the processes involved in the generation, acceleration and transport of solar energetic particles (SEPs) and to develop new modeling efforts to describe the physics at play in SEP events.

The continuous observation of SEP events by spacecraft located close to the Earth's orbit such as the WIND spacecraft, the Interplanetary Monitor Platform (IMP-8), the Geostationary Operational Environmental Satellites (GOES), the Advanced Composition Explorer (ACE), and the SOlar and Heliospheric Observatory (SOHO) has provided us with the required information to: (1) expand the statistical studies of SEP events at 1 AU (Reedy 2005), (2) analyze the possible correlations between the properties of the solar events and the parameters that characterize the SEP events (Kahler 2001; Kahler 2005), (3) identify the origin of the seed particle populations accelerated during large shock-associated SEP events (Desai et al. 2004), and (4) re-activate the modeling efforts to describe the processes of acceleration and transport of SEPs (Lee 2005 and references therein).

The conventional classification of SEP events distinguishes gradual and impulsive SEP events (Reames 1999). Impulsive events are rich in electrons, 3He and heavy ions with ionization states typical of a hot plasma $(\sim 5-10 \mathrm{MK})$ origin. Gradual events are protonrich, well associated with coronal mass ejections (CMEs), and have ion abundances near 
coronal values with ionization states typical of a 1-2 MK plasma. Under this paradigm, it is believed that impulsive events have their origin during rapid flares (lasting from a few minutes to an hour) whereas the processes of particle acceleration at the CMEdriven shocks dominate in gradual events. Narrow CMEs or jet-like ejecta have also been observed in association with the origin of impulsive events (Kahler 2001). Analyses of the elemental abundances measured during large, shock-associated SEP events have shown that these discriminators are in fact mixed. For example, CME-associated events are often enriched in $\mathrm{Fe} / \mathrm{O}$ ratios relative to nominal coronal values and show high ionization states that increase with increasing energy (Cohen et al. 1999; Mazur, et al. 1999). Furthermore, ${ }^{3} \mathrm{He}$ is enriched in a large fraction of shock-associated events (Desai et al. 2003). These new compositional studies have established that the origin of the SEPs accelerated by CME-driven shocks is not the solar wind as previously believed, but rather the suprathermal tail of the solar wind distribution (Gloeckler 2003), with possible additional contributions from either prior SEP events or from contiguous flare processes occurring at the Sun during the origin of the SEP events. Therefore, the terms "impulsive" and "gradual" do not clearly distinguish the origin of the particles. For a review of the origins and correct usage of the terms "impulsive" and "gradual" see, for example, Cliver \& Cane (2002).

The contribution of flare processes to the particle populations observed in large shockassociated SEP events is still under debate (Cane et al. 2003). Whereas some researchers consider that gradual events are the product solely of injection and acceleration of particles by CME-driven shocks from different source populations and with different shock geometries (Tylka et al. 2005 and references therein), other researchers argue for alternative SEP injection scenarios based on flares (Cane \& Erickson 2003) or additional flare/coronal phases of SEP injections that may occur at solar sites distant from the flaring parent active region (Klein et al. 2001; Klein et al. 2005).

Comparison between electromagnetic signatures associated with the flare processes and the arrival at $1 \mathrm{AU}$ of electrons with energies of tens of $\mathrm{keV}$ allowed us to infer that the injection of near-relativistic electrons back at the Sun is delayed, on average, by $\sim 10$ minutes from both the start of the type III radio burst emissions and the electromagnetic radio and X-ray signatures of the parent flare (Krucker et al. 1999; Haggerty $\&$ Roelof 2002). This delayed injection suggests that $\geqslant 20 \mathrm{keV}$ electrons are probably accelerated by a coronal or CME shock wave and not directly related to the electrons responsible for the solar electromagnetic radiations (Krucker et al. 1999, Krucker \& Lin 2002; Simnett et al. 2002). Alternatives to the late acceleration scenario include delay by transport processes in interplanetary space (Cane 2003) or as a result of the interplay between time-extended acceleration processes and particle injection into different magnetic structures (Klein et al. 2005).

The launch in 2002 of the Ramaty High Energy Solar Spectroscopic Imager (RHESSI) with its imaging and spectroscopic capabilities of both the hard X-ray/gamma-ray continuum and gamma ray lines emitted by energetic electrons and ions, respectively, has opened new possibilities for relating the SEPs observed in interplanetary space to the accelerated particle population interacting at the flare site (Lin 2005). RHESSI observations allow us to accurately locate the interacting particles at the Sun, providing information on the spectra of the parent electrons and ions as well as ion composition at the Sun. Early work on correlating the interplanetary and solar data sets provided indications of jet-like upward motion near the hard X-ray (HXR) emitting foot-point regions in some events associated with impulsive SEP events (Krucker et al. 2003; Krucker et al. 2004). For events observed above $50 \mathrm{keV}$ with a close temporal agreement between the HXR and the in-situ detected electrons (taking the time of flight of the escaping electrons into 
account), Krucker et al. (2005) found a correlation between the HXR photon spectral index and the electron spectral index of the in-situ electrons, indicating a common acceleration mechanism. In addition, the X-ray source structure for all these events appears to be similar, showing hot loops with HXR foot-points plus an additional HXR source separated from the loop. This structure can be explained by magnetic reconnection models with newly emerging flux tubes that reconnect with previously open field lines, thus allowing for the escape of energetic particles into the interplanetary medium.

As of September 2005, RHESSI has detected 14 gamma-ray line flares. Comparisons between the spectra of energetic protons producing gamma-ray lines at the Sun and the spectra of the energetic protons observed at $1 \mathrm{AU}$ are essential to understand the origin of, and the possible relationship between, both particle populations. Similarity between these spectra, if found, suggests that the gamma-rays and the insitu observed energetic protons may have a common source. On the other hand, the standard two-class paradigm completely separates the origin of the flare-accelerated and CME-shock-accelerated ions (Reames 2002). It is clear that RHESSI observations will help us to not only identify the sources of SEPs but also increase our understanding regarding the SEP acceleration processes occurring at the Sun.

Observations of ultra-heavy ions $(Z \geqslant 30)$ in some impulsive events (Reames 2000; Mason et al. 2004) have shown that the pattern of increasing abundance enhancements with increasing mass (or Z) extends to the top of the periodic table, with typical enhancement factors $>100$ for masses $\sim 200 \mathrm{amu}$, and reaching $>1000$ in some events. In addition, the enhancement pattern observed in these events is well organized as a power-law with respect to the mass-to-charge ratio of the elements if one assumes equilibrium charge states characteristic of a plasma at a few million degrees (Mason et al. 2004). Models based on plasma heating processes and/or stochastic particle acceleration in solar flares may explain the ${ }^{3} \mathrm{He}$-enrichment observed in these events (e.g., Liu et al. 2004), but fail to model the combination of heavy and ultra-heavy ion enrichments, charge states and electron associations usually observed in these events.

Advances in modeling large shock-associated SEP events have been described by Lario (2005), Lee (2005) and references therein. The multiple processes involved in the development of the SEP events include acceleration and transport of particles in a timedependent system formed by a propagating CME-driven shock, the associated evolving magnetic field topology both upstream and downstream of the shock, and the formation of magnetic field fluctuations (the last of which are also affected by the propagating particles). The difficulty of modeling all these processes is evident and many simplifying assumptions, not always well constrained by observations, are necessary. Increasingly detailed modeling of the evolving shock properties, wave-particle interactions and particle transport has been able to reproduce or predict major features of the shock-associated SEP events (Lario 2005). However, these models still include rough approximations such as the assumption of simplified magnetic field topologies both upstream and downstream of the traveling shocks, the existence of a stable and pre-determined medium where CMEs and particles propagate, the assumption that only one particle acceleration mechanism is at work in traveling shocks, the injection of only mono-energetic particle populations into the shock acceleration mechanisms, and the introduction of shocks at arbitrary $(\sim 10$ 20 solar radii) distances from the Sun, when there is strong evidence that particle acceleration may start closer to the Sun ( $\sim 2$ solar radii). An effort should be made in the following years to: (1) extend shock models to lower heights, (2) include seed-particle populations for the mechanisms of particle acceleration at shocks that contain both suprathermal tail solar wind populations and remnants from prior and contiguous events, (3) incorporate time variability in shock geometries and shock-acceleration mechanisms, and 
(4) consider realistic transport models in complex scenarios formed during the major SEP events that rarely occur in isolation. Examples of these compound series of events in solar cycle 23 were the Bastille Day 2000 event (Smith et al. 2001) and the October-November 2003 series of events (Lario 2005).

Spacecraft located beyond 1 AU such as Ulysses, Cassini and Voyager-2 have also allowed us to: (1) study the characteristics of SEP events at distances beyond 1 AU (Lario 2005) and high heliographic latitudes (Lario et al. 2003), (2) perform multi-spacecraft analyses of SEP events (Lario et al. 2000; McKibben et al. 2001), (3) relate simultaneous observations of major SEP events by distant spacecraft to either heliospheric transport processes (McKibben et al. 2003) or to the characteristics of parent solar events (Lario et al. 2003), and (4) use energetic particle observations to determine the largescale structure of the heliosphere and the conditions for particle transport throughout the interplanetary medium (Lario et al. 2004).

In particular, observations of SEP events by Ulysses during its solar maximum orbit above the poles of the Sun have allowed us to conclude that: (1) major SEP events are simultaneously observed by Ulysses and Earth-orbiting spacecraft regardless of the longitudinal, latitudinal and radial separation between the observers (Lario et al. 2000, Lario et al. 2003; McKibben et al. 2001, McKibben et al. 2003), (2) particle anisotropy flows are aligned with the magnetic field indicating that no net flow across the local magnetic field is observed (Sanderson et al. 2003), and (3) the decay phase of the major SEP events as observed from distant inner heliospheric locations evolves with comparable particle intensities and similar decay-rates suggesting the existence of a region with zero radial, longitudinal and latitudinal particle intensity gradients (McKibben et al. 2003). Implications of these observations for the particle transport and the large-scale structure of the inner heliosphere are described elsewhere (e.g., Lario et al. 2003). Several of these major events of solar cycle 23 have also been observed at large heliocentric distances by the Voyager-2 spacecraft in association with the passage of merged interaction regions (MIRs) formed by the coalescence of multiple CMEs en route to the outer heliosphere (Decker \& Krimigis 2002; Lario 2005).

\section{References}

Cane, H.V. 2003, ApJ 598, 1403-1408

Cane, H. V. \& W. C. Erickson 2003, JGR 108, doi:10.1029/2002JA009488

Cane, H. V., T. T. von Rosenvinge, C. M. S. Cohen \& R. A. Mewaldt 2003, GRL 30, doi;10.1029/2002GL016580

Cliver, E. W. and H. V. Cane 2002, EOS Trans. AGU 83, 61-68

Cohen, C. M. S. et al. 1999, GRL 26, 2697-3000

Decker, R. B. \& S. M. Krimigis 2003, Adv. Space Res. 32, 597-602

Desai, M. I. et al. 2003, ApJ 588, 1149-1162

Desai, M. I. et al. 2004, ApJ 611, 1156-1174

Gloeckler, G. 2003, in: M. Velli et al. (eds.), Solar Wind Ten CP679, 583-588

Haggerty, D. K. \& E. C. Roelof 2002, ApJ 579, 841-853

Kahler, S. W. 2001, JGR 106, 20947-20955

Kahler, S. W. 2005, ApJ 628, 1014-1022

Kahler, S.W., D.V. Reames \& N.R. Sheeley 2001, ApJ 562, 558-565

Klein, K.-L., G. Trottet, P. Lantos \& J.-P. Delaboudiniere 2001, A\& A 373, 1073-1082

Klein, K.-L., S. Krucker, G. Trottet \& S. Hoang 2005, A\&A 431, 1047-1060

Krucker, S., D. E. Larson, B. P. Johnson \& R. P. Lin 1999, ApJ 519, 864-875

Krucker, S. \& R. P. Lin 2002, Solar Phys 210, 229-243

Krucker, S. et al. 2003, EOS Trans. AGU 84(46), Fall Meet. Suppl., Abstract SH11D-1130 
Krucker, S., R. P. Lin \& E. P. Kontar 2004, EOS Trans. AGU 85(17), Jt. Assem. Suppl., Abstract SH22A-02

Krucker, S., E. P. Kontar \& R. P. Lin 2005, in preparation

Lario, D. 2005, Adv. Space Res. doi:10.1016/j.asr.2005.07.081, in press

Lario, D. et al. 2000, JGR 105, 18251-18274

Lario, D., E. C. Roelof, R. B. Decker \& D. B. Reisenfeld 2003, Adv. Space Res. 32, 579-584

Lario, D. et al. 2004, JGR 109, A09S02, doi:10.1029/2003JA010107

Lario, D. et al. 2005, JGR 110, A09S11, doi:10.1029/2004JA010940

Lee, M.A. 2005, ApJ Suppl. Ser. 158, 38-67

Lin, R. P. 2005, Adv. Space Res. 35, 1857-1863

Liu, S., V. Petrosian \& G.M. Mason 2004, ApJ 613, L81-L84

Mason, G. M. et al. 2004, ApJ 606, 555-564

Mazur, J. E. et al. 1999, GRL 26, 173-176

McKibben, R. B. et al. 2001, in Proc. 27th Int. Cosmic Ray Conf. 3281-3284

McKibben, R. B. et al. 2003, Ann. Geophys. 21, 1217-1228

Reames, D. V. 1999, Space Sci. Rev. 90, 413-491

Reames, D. V. 2000, ApJ 540, L111-L114

Reames, D. V. 2002, ApJ 571, L63-L66

Reedy, R. C. 2005, Lunar and Planetary Science XXXVI abstract \#2184

Sanderson, T. R. et al. 2003, GRL 30, 8036, doi:10.1029/2003GL017306

Simnett, G. M., E. C. Roelof \& D. K. Haggerty 2002, ApJ 579, 854-862

Smith, C. W. et al. 2001, Solar Phys. 204, 227-252

Tylka, A. J. et al. 2005, ApJ 625, 474-495

\section{Heliospheric Compositional Signature}

Giannina Poletto

(INAF, Arcetri Astrophysical Observatory, Firenze Italy; poletto@arcetri.astro.it)

\subsection{Introduction}

Remote observations of the solar elemental composition reveal variations in the element abundances, that depend on the site, or on the phenomenon, where abundances are measured. Because in situ measurements also show changes in the composition of plasma, the study of elemental composition is a powerful tool for identifying the source region of plasma observed in situ. Here I will focus on the heliospherical signatures of slow vs. fast wind and of Coronal Mass Ejections (CMEs). As we will see in the following, charge state compositional signatures are a further means to establish the connection between remotely observed and in situ events. Controversial results, some mentioned hereafter, may depend on which part of the event is being sampled by in situ instrumentation: an often neglected factor that should be taken into account

\subsection{Slow and fast solar wind}

At minimum solar activity, when the solar atmosphere is composed of large coronal holes (CHs) at high latitudes and active regions (ARs), if any, in the equatorial belt, Ulysses observations show a clear distinction between low latitude slow and fast polar wind. Near the maximum phase, there is no longer evidence for a bimodal nature of solar wind and the need for a tool to identify the source region of the wind becomes more stringent (McComas et al. 2002).

Elemental composition provides one of the means to relate coronal and in situ measurements. The latter show that in slow wind elements with a low First Ionization Potential $($ FIP $\leqslant 10 \mathrm{eV})$ are $\approx$ a factor $3-4$ (FIP bias) more abundant than they are in the photosphere, while in fast wind abundances are nearly photospheric. Because the elemental 
composition is dictated by processes in the low solar atmosphere, differences in elemental composition of fast and slow wind reveal their sources to be located in different solar regions. Traditionally, the remotely observed photospheric abundances of CHs have been taken as a further demonstration that high speed wind emanates from CHs. Assuming this to be a valid conclusion, there is still controversy on whether plumes or interplume regions should be considered as sources of fast wind (Teriaca et al. 2003; Gabriel et al. 2003). Abundances at the base of polar plumes have been derived by Del Zanna et al. (2003) who found them to be close to photospheric values, in contrast with previous results. In situ measurements have not been able, yet, to discriminate between plume and interplume abundances. This is an area where further work is necessary.

The reason for the lack of enrichment in low-FIP elements in coronal holes is controversial as well. Wang \& Sheeley (2003) interpret it within the traditional scenario where the wind speed depends on the expansion geometry of $\mathrm{CHs}$, but a different view is suggested by Woo et al. (2004), who claim the FIP bias is dictated by the duration of plasma confinement in solar loops. As a consequence, a low FIP bias indicates a rapid release of elements from the loops where they were originally trapped and the fast, low FIP wind from high latitudes implies that those loops have not been able to hold elements long enough to enrich their abundances. There are no definitive arguments in favor of either proposal.

In situ abundances of slow wind are greatly variable, as opposed to the stability of abundances in fast wind. An attempt to compare coronal values of the ratio $\mathrm{Fe} / \mathrm{O}$ (a proxy for the FIP effect) with in situ Fe/O values of the same plasma has been made by Bemporad et al. (2003), taking advantage of the SOHO/Ulysses quadrature geometry. Although remote and in situ values are in good agreement, Bemporad et al. point out that the large fluctuations in in situ measurements allow only for a comparison between time-averaged in situ values and coronal values.

Beside the short term variability, the strength of the fractionation effect depends on heliographic latitude (von Steiger et al. 2002) and on the phase of the solar cycle (Zurbuchen et al. 2002). Both phenomena have an explanation in the Fisk (2003) model, which invokes frequent reconnection between closed and open loops. Because in this model the FIP bias depends on parameters like the length and age of the loops, the FIP variability would provide information on the behavior of loops with latitude and solar cycle. The capability of accounting for coronal and in situ observations within an unified framework makes the Fisk model very appealing: establishing the dependence of coronal abundances on loop parameters will put the proposed scenario on more solid ground.

At solar maximum active regions are a further source of solar wind (Woo \& Habbal 2005). ARs responsible for solar wind outflows may be identified by tracing back to their coronal source the wind streams observed in situ via a ballistic and a potential field source surface model (see, e.g., Neugebauer et al. 2002). These authors use charge state ratios, rather than element abundances, to define differences between wind from $\mathrm{CHs}$ and from ARs; the latter typically showing a higher and more variable $O^{7+} / O^{6+}$ ratio (Liewer et al. 2004). Recently it has been shown that the $O^{7+} / O^{6+}$ ratio correlate inversely with the chromospheric "depth" evaluated from TRACE data (McIntosh \& Leamon 2005). This result indicates that the solar wind may be rooted much more deeply in the solar atmosphere than assumed so far.

\subsection{ICME signatures}

Charge state ratios, together with helium abundances and composition anomalies, have been used as a means to identify the interplanetary counterparts (ICMEs) of CMEs. These are among the many signatures of ICMEs (see, e.g., Zurbuchen \& Richardson 
2005)) which, however, do not occur at the same time, do not show up in all events, and may vary depending on the nature of the ICMEs. According to Richardson \& Cane (2004) both magnetic clouds and non-magnetic clouds have higher charge state ratios and higher FIP bias than the ambient wind (but magnetic clouds values tend to be less variable than they are in other ICMEs). This finding contradicts previous results (Henke et al. 2001) which claimed that non-cloud structures have the same ionization state as their source region (ARs or $\mathrm{CHs}$ ). This controversy points to the need for further analysis of this issue.

Fe charge states $\geqslant 16$ have been often observed in situ, as opposed to an average charge state $\approx 12$. These higher charge states are considered to be a further signature of ICMEs: the coronal source responsible for the highly ionized Fe observed in one such event has been unambiguously identified by Poletto et al. (2004) in the current sheet formed in the aftermath of a CME. The variation of the high Fe charge states measured in situ with latitude and phase of the solar cycle has been studied (Lepri \& Zurbuchen 2004) and interpreted in terms of different magnetic connectivity to flaring regions. Higher charge states of $\mathrm{Fe}$, as well as higher values of the $O^{7+} / O^{6+}$ ratio, have been found by Reinard (2005) to be moderately correlated with flare magnitude, thus confirming that enhanced charge states are related with flare-like heating.

The NASA Genesis mission has collected solar wind over more than two years. Samples returned to Earth in September 2004 and are being analyzed. An area where we expect advancement from the mission is in the measurements of the $\mathrm{He} / \mathrm{H}$ abundances made by the Genesis Ion Monitor (GIM). An alpha to proton ratio $\geqslant 8 \%$ is used as a criterion for the presence of an ICME, although is not clear at all whether this implies an high alpha to proton ratio also in the corona (Zurbuchen et al. 2003). First results from Genesis are being presented (Reisenfeld et al. 2005) in terms of the recently revised solar elemental abundances (Asplund et al. 2005) and seem to point to larger He enhancements in faster ICMEs.

This brief outline of advancements in this area is necessarily incomplete. The interested reader is referred to the excellent reviews that have appeared recently in the literature, such as Richardson \& Cane (2004), Feldman et al. (2005), and von Steiger \& Zurbuchen (2003).

\section{References}

Asplund, M., Grevesse, N., Sauval, A. J. 2005, in F. N. Bash \& Barnes, T. G. (eds.) Cosmic Abundances as Records of Stellar Evolution and Nucleosynthesis, Vol. XXX, in press

Bemporad, A., Poletto, G., Suess, S. T., Ko, Y.-K., Parenti, S., Riley, P., Romoli, M. \& Zurbuchen, T. 2003, ApJ 593, 1146

Del Zanna, G., Bromage, B.J.I. \& Mason, H.E. 2003, AA 398, 743

Feldman, U., Landi, E. \& Schwadron, N. A. 2005, JGR, 110, A07109, doi:10.1029/2004JA010918

Liewer, P. C., Neugebauer, M., Zurbuchen, T. 2004, SPh 223, 209

Lepri, S. T. \& Zurbuchen, T. H. 2004, JGR, 109, (A1), 1112

Linton, C.M. \& Evans, D.V. 1992, Phil. Trans. R. Soc. Lond. A338, 325

McComas, D. J., Elliott, H. A., Gosling, J. T., Reisenfeld, D. B., Skoug, R. M., Goldstein, B. E., Neugebauer, M. \& Balogh, A. 2002, GRL 29(9), 10.129/2001GL014164

McIntosh, S. W. \& Leamon, R. J. 2005, ApJ, 624, L117

Neugebauer, M., Liewer, P. C., Smith, E. J., Skoug, R. M. \& Zurbuchen, T. H. 2002, JGR 107, (A12), SSH 13-1

Poletto, G., Suess, S. T., Bemporad, A., Schwadron, Elliott, H. A., Zurbuchen, T. H. \& Ko, Y.-K. 2004, ApJ, 613, L173

Reinard, A. 2005, ApJ, 620, 501 
Reisenfeld, D. B., Wiens, R. C., Barraclough, B. L., Steinberg, J. E., DeKoning, C., Zurbuchen, T. \& Burnett, D. S. 2005, in Lunar and Planetary Science XXXVI, abstract 1278

Richardson, I. G. \& Cane, H. V. 2004, JGR, 109(9), doi:10129/2004JA010598

Riley, P., Linker, J. A., Mikic, Z., Odstrcil, D., Webb, D. F. \& Zurbuchen, T. H. 2004, EOS

Trans. AGU 84(46), Fall Meet. Suppl., abstract SH24A-07

von Steiger, R. 2002, Cospar Scientific Assembly, Houston 2002

von Steiger, R. \& Zurbuchen, T. H. 2003, in A. Wilson (ed.) Solar Variability as an Input to the Earth's Environment, SP-535, ESA, ESTEC, Noordwijk, The Netherlands, p. 835

Zurbuchen, T. H. \& Richardson, I. G. 2005, Space Sci. Rev., in press

Zurbuchen, T. H., Fisk, L. A., Gloeckler, G. \& Von Steiger, R. 2002, Geophys. Res. Let. 29, 9, 66

Zurbuchen, T. H., Fisk, L. A., Lepri, S. T. \& Von Steiger, R. 2003, in M. Velli, R. Bruno, F. Malara (eds.), Solar Wind Ten (AIP), p. 604

Wang, Y.-M. \& Sheeley, N. R. 2003, ApJ 587, 818

Woo, R., Habbal, S. R. \& Feldman, U. 2004, ApJ Lett 612, L1171

Woo, R., Habbal, S. R. 2005, ApJ Lett 629, L129

\section{Ulysses Observations and Solar Wind Pickup Ions}

Steven Suess

(NASA Marshall Space Flight Center, National Space Science \& Technology Center/Solar Physics, Huntsville, Alabama; Steven.T.Suess@nasa.gov)

\subsection{Introduction}

Neutral atoms that are ionized and incorporated into the solar wind are known as pickup ions. They have many sources, including interstellar gas entering the heliosphere, dust, and comets, and other sources as yet unknown. Atoms may travel great distances before they are ionized and, thus, the study of pickup ions allows analysis of processes and sources far removed from the observer. The presence of pickup ions is determined by measurements of the solar wind ion distribution function. Measurement of the spatial and temporal gradients of pickup ions separates individual sources (Schwadron 2004) and distinguishes them from other composition and ionization state signatures, such as those of coronal mass ejections (CMEs) and fast versus slow wind (the 'FIP effect') (Bemporad et al. 2005, Balogh et al. 2001).

The Ulysses Solar Wind Ionization and Composition Spectrometer (SWICS) routinely detects pickup ions and has been in continuous operation since shortly after launch in 1990. Ulysses passed Jupiter in early 1992 to enter a solar polar orbit of inclination $80.2^{\circ}$, perihelion 1.34 AU, aphelion 5.4 AU, and period 6.2 years. From this orbit, Ulysses has been able to discover and characterize many properties of various pickup ions populations. Since an orbit lasts for a substantial portion of a solar sunspot cycle, the spatio-temporal cuts through the heliosphere sometimes cannot isolate particular sources. However, an instrument identical to SWICS is on the ACE spacecraft at the L1 point, and similar measurements are made on MESSENGER and Cassini (and will be made on STEREO after its launch in 2006). The distributed in-ecliptic measurements, not in existence during the Ulysses measurements at the last solar minimum, will enable the isolation and determination of most sources and their properties during the upcoming 2007-2008 solar minimum.

\subsection{Local interstellar cloud}

Local interstellar cloud pickup ions (LICPIs) of N, Ne, O, H, He are routinely detected by SWICS (Gloeckler et al. 2001, Gloeckler \& Geiss 2004). These ions, when carried by the solar wind out to the termination shock (TS), are accelerated at the TS to contribute 
to the anomalous cosmic ray (ACR) population that is observed in the inner heliosphere. In the last few years, Ulysses measurements of LICP $\mathrm{H}^{+}$played an important role in correctly predicting the crossing of the TS by Voyager 1 in late 2004 (Fisk 2005).

Studies of velocity distributions upstream and downstream of shocks using Ulysses SWICS and HISCALE data, made it possible to construct $\mathrm{H}^{+}$differential energy spectra upstream and downstream of the TS (Gloeckler et al. 2005). Contrary to general expectations that the observed TS spectra result from diffusive shock acceleration, it was found that simply heating the upstream distributions of pickup and solar wind ions by an amount given by the Rankine-Hugoniot relations for a gas dynamic shock gives excellent fits to the measured ion spectra and is consistent with all other available Voyager 1 observations. These measurements, along with those being made by Voyagers 1 and 2, determine the acceleration efficiencies for ACRs. SWICS abundances of LICPIs and the abundances of corresponding elements in the solar wind are being combined with Voyager 1 and 2 composition measurements of low energy $(\sim 0.5$ to $\sim 3 \mathrm{MeV} /$ nuc) particles at the TS to establish the relative contribution of solar wind and LICPIs to the low energy particles accelerated by the TS to become ACRs.

In another study, measurements with the Ulysses/GAS instrument of neutral He and with SWICS and ACE of pickup $\mathrm{He}^{+}$and $\mathrm{He}^{++}$were central in a multi-spacecraft coordinated effort to determine the best consistent values for the physical parameters of the LIC gas based on particle and UV observations (Möbius et al. 2004). Using the spatiotemporal information from Ulysses, together with Ulysses, Cassini, ACE, and MESSENGER measurements of interstellar $\mathrm{H}^{+}$and $\mathrm{He}^{+}$, it is possible to determine the spatial and temporal variations of ionization rates for $\mathrm{H}$ and $\mathrm{He}$ and to determine whether there are density inhomogeneities in the LIC.

\subsection{Dust}

Inner source pickup ions (ISPIs) are singly ionized with velocity distributions that peak at or below the solar wind speed and a composition of the most abundant elements that is similar to the solar wind. Their intensities decrease roughly with inverse heliocentric distance, implying a source near the Sun. Latitudinal and solar cycle variations have been discovered but are not understood.

The origin of ISPIs remains an open question. One scenario (Schwadron et al. 2000) is that ISPIs originate as solar wind material that is imbedded in dust grains close $\left(\sim 10-30 R_{\odot}\right)$ to the Sun and then released as slow moving atoms and molecules that are subsequently ionized and picked up by the solar wind. This can be determined by additional measurements near solar minimum with the help of ACE, MESSENGER, and STEREO. These measurements also have the potential of determining the spatial distribution and solar cycle variations of the dust in the vicinity of the Sun through which Solar Probe will fly.

\subsection{Comets}

As a comet approaches the Sun it emits at an increasing rate volatile material consisting mainly of water group molecules. This neutral gas expands at typical speeds of $\sim 1 \mathrm{~km} / \mathrm{s}$. It is quickly dissociated and ionized, to be picked up and swept away from the Sun and to form a thin, long ion tail that extends radially outward to distances of at least several AU. The pickup ions have a characteristic velocity spectrum with a sharp drop in density at a well defined cutoff speed which is related to the radial distance from where the gas was first ionized to the location where the pickup ions are observed.

Direct sampling and remote sensing have established the characteristic composition of cometary gas (von Rosenvinge et al. 1986, Nature 1986, Crovisier \& Bockelée-Morvan 
1999, Huebner \& Benkhoff 1999). Direct sampling generally requires spacecraft close to comets. Nevertheless, two unplanned, distant crossings of cometary tails by Ulysses have shown that it is possible to sample cometary pickup ions at large radial and angular separation from the comet, using the characteristic composition for identification.

The first unexpected comet tail crossing occurred in 1996 during a near-radial alignment with comet Hyakutake at $0.35 \mathrm{AU}$ when Ulysses was at $3.7 \mathrm{AU}$ (Gloeckler et al. 2000, Jones et al. 2000). The radial separation was large in this case.

The second unexpected Ulysses comet tail crossing was with comet McNaught-Hartley, and perhaps comet C/2000 S5. Unlike the first crossing, this was at large angular separation from Ulysses (Gloeckler et al. 2004). This detection was possible due to a CME that distorted the heliospheric magnetic field sufficiently to guide pickup ions produced near $(\lesssim 1$ million $\mathrm{km}$ ) the comets to Ulysses located more than 150 million $\mathrm{km}$ from the comets. Such distortions, both transient and long-lasting, are known as magnetic "deviations" from the classical spiral magnetic field.

The ability of CMEs to carry cometary ions far from their radial paths significantly increases the probability of detecting these ions. In many respects, the first unplanned comet-tail crossing by Ulysses was seen as an anomaly. However, a second crossing shows that chance detection of comet tails is more likely than thought. Clearly, the presence of CMEs increases the odds of an event.

\subsection{Prospects}

The study of pickup ions is about connections. It requires an understanding of how in situ ions and energetic particles are transported over time and space. It permits diagnosis of conditions at locations in and near the heliosphere at otherwise presently unreachable locations through the interpretation of connections between local measurements and distant sources. New measurements with spatially distributed instruments during the next solar minimum will greatly expand the results from these studies.

\section{References}

Balogh, A., R. G. Marsden, \& E. J. Smith 2001, in A. Balogh, R. G. Marsden, \& E. J. Smith (eds), The Heliosphere Near Solar Minimum: The Ulysses Perspective, Praxis Publishing Ltd

Bemporad, A., G. Poletto, S. T. Suess, Y.-K. Ko, N. A. Schwadron, H. A. Elliott, \& J. C. Raymond 2005, ApJ, in press

Crovisier, J. and Bockelée-Morvan, D. 1999, Space Sci. Rev., 90, 19

Fisk, L. A. 2005, Science, 309, 2016

Gloeckler, G. et al. 2000, Nature, 404, 576

Gloeckler, G., J. Geiss, \& L. A. Fisk, 2001, in A. Balogh, R. G. Marsden, \& E. J. Smith (eds), The Heliosphere Near Solar Minimum: The Ulysses Perspective, 287, Praxis Publishing Ltd

Gloeckler, G., \& J. Geiss 2004, Adv. Space Res., 34, 53

Gloeckler, G., Allegrini, F., Elliott, H. A., McComas, D. J., Schwadron, N. A., Geiss, J., von Steiger, R., \& Jones, G. H. 2004, Ap. J. Lett., 604, L121

Gloeckler, G., L. A. Fisk, \& L. J. Lanzerotti 2005, in Solar Wind 11, ESA, in press

Huebner, W. F. and Benkhoff, J. 1999, Space Sci. Rev., 90, 117

Jones, G. H., Balogh, A., and Horbury, T. S. 2000, Nature, 404, 574

Möbius, E. et al. 2004, A\&A, 426, 897

Nature, Encounters with comet halley: the first results, 1986, Nature (Suppl.), 321, 259

Schwadron, N. A., J. Geiss, L. A. Fisk, G. Gloeckler, T. H. Zurbuchen, \& R. von Steiger 2000, $J G R, 105,7465$ 
Schwadron, N. 2004, in: G. Poletto \& S. T. Suess (eds.), The Sun and the Heliosphere as an Integrated System, p. 179, Kluwer Academic Publishers

von Rosenvinge, T. T., Brandt, J. C., and Farquhar, R. W. 1986, Science, 232, 353

\section{The Termination Shock and Heliospheric Boundary}

Rosine Lallement

(Service d' Aeronomie du CNRS, Verrieres-le-Buisson, France;

Rosine.Lallement@aerov.jussieu.fr)

\subsection{Introduction}

The last three years have been exceptional for the heliospheric community: in December 2004 Voyager 1 crossed the solar wind termination shock, the inner boundary of our heliosphere, becoming the first spacecraft to begin exploring the heliosheath. Magnetic field and low energy cosmic ray spectra both imply a shock strength of $\mathrm{r}=2.4-3.0$. This crossing opens up a new era; Voyager 1 will gather a continuous flow of unprecedented data from the heliosheath on its way to the heliopause. There are already some surprises concerning the energy distribution of accelerated particles and the location of acceleration.

Apart from this historic event, new measurements of the interstellar neutral hydrogen and helium flow directions have revealed a few degrees difference. This has been interpreted as a deviation of the $\mathrm{H}$ flow due to charge-transfer coupling to a tilted heliospheric interface, such as the one resulting from a non-axial interstellar magnetic field. New models reproduce this deviation.

Two results may have implications in other research fields. The first one is the significant contribution of the heliosphere to the diffuse X-ray background. The emission, due to charge transfer between solar wind ions and neutrals, has been shown to be of the same order as the thermal X-ray emission from 50-150 parsecs of hot and ionized interstellar gas. It contaminates the emission of faint distant astronomical objects, as demonstrated in the case of an XMM observation of the Hubble Deep Field-North. On the other hand, the composition of the local interstellar medium deduced from pickup ions has been interpreted as the sign of a local infall of extra-galactic material.

Finally, a new space mission, the Interstellar Boundary Explorer has been selected by NASA. IBEX will provide energetic neutral atoms (ENA) images of the heliosphere.

\subsection{Constraints on the heliospheric boundary}

Models of the heliospheric interface predict the supersonic-subsonic transition of the solar wind at the so-called termination shock (TS), the innermost transition, a further contact discontinuity, the heliopause (HP) and a bow shock that is supposed to decelerate the interstellar plasma before reaching the heliopause. Interstellar charged particles are excluded from the heliosphere while neutral atoms enter it.

The latest models all predicted a distance to the solar wind termination shock in the range 90-105 A.U. Such a quantitative description was based on a number of observations providing interstellar parameters or distances to the discontinuities.

(i) The solar wind data of Voyager 2, now at 77 A.U (mid-2005) have been compared with model predictions based on data at 1 A.U. There is a clear departure between the predicted free expansion average velocity and the observed one, due to interstellar neutral mass loading (atoms getting ionized become pickup ions which are convected outwards in the solar wind). The measured deceleration has been modeled to infer the neutral hydrogen density at the termination shock. The value found for solar maximum, i.e. 
$0.09 \mathrm{~cm}^{-3}$ (Wang \& Richardson 2003), is in rather good agreement with other results, mainly pickup ion data and backscattered Lyman-alpha radiation. The Voyager data have also been used to predict the motions of the termination shock back and forth under the action of enhanced and decreasing solar wind pressure. In the light of the latest results and the shock crossing, Richardson et al. (2005) show that Voyager 1 was actually very close to the shock in mid-2003, but did not cross it as the shock started to move away faster than the spacecraft velocity under the effect of increased solar wind pressure. The crossing occurred in December 2004 when the shock moved back again.

(ii) Radial gradients, energy spectra and time variations of modulated galactic cosmic rays (GCR) and anomalous cosmic rays (pickup ions accelerated in the outer heliosphere) have been used in different ways to infer the distance to the shock and the shock motion completely independently of any interstellar parameter. Stone \& Cummings (2003) in particular showed that in 2002 (Voyager 1 at 84 A.U.) the shock was closer than 92 A.U.

(iii) Radio waves in the $2-3 \mathrm{kHz}$ range have been detected after solar maximum for the last three cycles. These emissions are very likely generated at the heliopause (HP) when it is hit by particularly intense solar wind events. The time delay between the mass ejections and the emissions allows an estimate of the distance to the heliopause. Using their latest data, Gurnett \& Kurth (2005) predicted d(HP) $=153-158$ A.U. Combined with model results, this HP distance places the TS at 101-108 A.U.

(iv) Interstellar hydrogen and helium atoms penetrate the heliosphere and bring complementary information. Helium atoms are extremely weakly coupled to the protons, and for this reason helium does not feel the heliospheric interface. It is an excellent tracer of interstellar conditions outside the heliosphere. Neutral hydrogen is strongly coupled to the protons through charge-transfer reactions, and a significant fraction of the $\mathrm{H}$ atoms entering the inner heliosphere are former interstellar protons having already experienced deceleration, heating and deflection around the heliosphere. Differences between $\mathrm{H}$ and He provide a measurement of the interstellar plasma, i.e., of what is actually confining the heliosphere. Recent results on neutral flow characteristics come from: 1) remote sensing of the solar radiation backscattered by He atoms (EUVE data, Vallerga et al. (2004)) and $\mathrm{H}$ atoms (hydrogen absorption cell data from the SOHO-SWAN experiment, (Lallement et al. 2005), 2) direct in situ measurements of neutrals (ULYSSES-GAS, Witte 2004), and 3) pickup ions distributions (ULYSSES-SWICS, ACE-SWICS, Gloeckler et al. 2004). Large datasets on neutral helium have been combined with solar data, narrowing the ranges for the parameters of the neutral helium flow (Möbius et al. 2004). On the other hand, SWAN data have been analyzed in detail, providing the most accurate measurements of the velocity, temperature and direction of the $\mathrm{H}$ flow. Using the density measurements and the $40 \%$ ionization of helium in the local interstellar medium deduced by Wolff et al. (1999), Izmodenov et al. (2003) derived a proton density of 0.04$0.07 \mathrm{~cm}^{-3}$, leading to a TS between 95 and 100 A.U. Using updated pickup ion data and the same model results, Gloeckler et al. (2004) predicted a TS crossing between 2003 and 2009.

The comparison between the He and $\mathrm{H}$ flow directions has revealed for the first time a 4 degree angular deviation of the $\mathrm{H}$ flow. This deflection of $\mathrm{H}$ at its entrance into the heliosphere is very likely due to charge-transfer with a non axial-symmetric plasma interface under the influence of an inclined interstellar magnetic field (Lallement et al. 2005). The heliosphere can be used as an interstellar magnetic compass, since the direction of the field is inferred from the respective directions of the $\mathrm{H}$ and He flows. A recent self-consistent, 3-D Kinetic-MHD model reproduces well the amplitude of the deviation for an interstellar magnetic field of $2.5 \mu$ Gauss making a $45 \mathrm{deg}$ angle with the flow 
(Izmodenov et al. 2005). A last missing parameter for the description of the heliosphere is now provided.

\subsection{Voyager 1 across the termination shock: the close approach in 2002 and the actual crossing in 2004}

Voyager 1 is now the most distant spacecraft from Earth since it passed Pioneer a few years ago. During the period August 2002-February 2003 intense beams of energetic ions and electrons, very similar to the expected precursors from the heliospheric termination shock, have been detected (Krimigis et al. 2003). At the same time, however, magnetometers did not measure the field compression which, in principle, is a strong characteristic of the shock (Burlaga et al. 2003), and the low-intensity level and spectral energy distribution of the anomalous cosmic rays indicated that Voyager 1 had not reached the termination shock (McDonald et al. 2003).

As detailed in Decker et al. (2005), and in the light of what happened later, the spacecraft had been actually traveling extremely close to, but upstream of the shock. Due to solar wind pressure variations however, the shock had been moving away from the Sun between 2003 and 2004.5, adding almost two more years to the cruise time within the supersonic solar wind.

The actual shock crossing took place on December 16, 2004 at 94 A.U.; there is now full consensus about that particular event. The magnetic field intensity jumped from $0.136 \pm$ $0.035 \mathrm{nT}$ by a factor $3.05 \pm 0.04$ (Burlaga et al. 2005). In December 2004 Voyager 1 observed intensity spikes of ions and electrons that were followed by a sustained factor of 10 increase at the lowest energies and lesser increases at higher energies. A smooth continuous increase followed. The azimuthal streaming index decreased and the variability strongly decreased, in agreement with models predicting a much smaller streaming anisotropy in the heliosheath (Stone et al. 2005). Since the crossing both ACRs and GCRs have risen smoothly. The new feature is the presence of a low energy population (protons $\leqslant 3.5 \mathrm{MeV}$ ), probably quickly accelerated within a thin (AU size) region around the shock, and clearly distinct from the ACRs (E up to $100 \mathrm{MeV}$ ). The spectral slope of the low energy particles implies that the TS is a weak shock with a shock strength $\mathrm{r}=2.6(+0.4-0.2)$, if as is likely, diffusive shock acceleration is the main process. This is in good agreement with the strength derived from the magnetometers. There is still an unexplained substantial modulation of the He and O ACRs at the location of the shock, and the anomalous helium (most part of the 27-69 MeV Helium particles) continues to increase after the shock (Stone et al. 2005). This is in agreement with Webber \& Lockwood (2004) who inferred from a large data set prior to the crossing the existence of a large modulation area beyond 95 A.U. These results may lead to revisions of acceleration models. Finally, electron plasma oscillations have been observed by the plasma wave instrument since February 2004 at 91 A.U. and until the crossing on December 2004, with a gradually increasing occurrence rate (Gurnett \& Kurth 2005). Since then, no further oscillations have been observed, which is consistent with the spacecraft having crossed the termination shock, and with the most likely origin for these waves, i.e., electron plasma oscillations driven upstream from the TS by an energetic electron beam from the shock itself.

More information is expected from both the Voyager 2 TS crossing, expected within the next decade, and the continuous and unprecedented data Voyager 1 will now record in the heliosheath. Hopefully the newly selected IBEX mission, which will detect energetic neutral atoms created after charge transfer between neutrals and thermal or supra-thermal solar wind ions in the heliosheath, will simultaneously bring new constraints (McComas et al. 2004). 


\subsection{Heliosphere and local interstellar medium}

The heliospheric diffuse X-ray background is due to radiative cascades of solar wind high ions after they have captured electrons from interstellar atoms, a phenomenon discovered around comets. This charge-transfer X-ray emission has been found to be of the same order as the diffuse emission from the so-called Local Bubble, a 50-150 parsecs wide region around the Sun filled with hot gas (Robertson \& Cravens 2003, Lallement 2004). It is time variable due to the solar wind inhomogeneity and depends on the observer location and the solar cycle phase. For faint and diffuse astronomical X-ray sources this emission may contaminate significantly the X-ray spectra below $1.5 \mathrm{keV}$. This has been demonstrated in the exemplary case of a long duration XMM-Newton exposure toward the Hubble Deep Field-North (Snowden et al. 2004).

A number of interstellar species have been detected in the heliosphere in the form of pickup ions which are routinely detected by the SWICS instruments on board Ulysses and ACE. Using abundances of ${ }^{3} \mathrm{He}, \mathrm{O}$ and N Geiss et al. (2002) suggest that $20-25 \%$ of the local interstellar gas is extra-galactic and results from the infall of matter processed in dwarf galaxies, i.e. more moderately processed by stellar nucleosynthesis than in the galactic disk.

\section{References}

Burlaga, L. F., Ness, N. F., Stone, E. C., et al. 2003, Geophys. Res. Letters, 30, 9

Burlaga, L. F., Ness, N. F., Acuña, M. H., et al. 2005, Science, 309, 2027

Decker, R. B., Krimigis, S. M., Roelof, E. C., et al. 2005, Science, 309, 2020

Geiss, J., Gloeckler, G., \& Charbonnel, C. 2002, ApJ, 578, 862

Gloeckler, G., et al. 2004, $A \mathscr{E} A, 426,845$

Gurnett, D. A., Kurth, W. S., \& Stone, E. C. 2003, Geophys. Res. Letters, 30, 8

Gurnett, D. A., \& Kurth, W. S. 2005, Science, 309, 2025

Izmodenov, V., Gloeckler, G., \& Malama, Y. 2003, Geophys. Res. Let., 30, 3

Izmodenov, V., Alexashov, D., \& Myasnikov, A. 2005, A\& A, 437, L35

Krimigis, S. M., Decker, R. B., Hill, et al, 2003, Nature, 426, 45

Lallement, R. 2004, A\&A, 418, 143

Lallement, R., Quémerais, E., Bertaux, et al, R. 2005, Science, 307, 1447

McComas, D., et al. 2004, AIP Conf. Proc., 719, 162

McDonald, F. B., Stone, E. C., Cummings, A. C., et al 2003, Nature, 426, 48

Möbius, E. et al. 2004, A\&SA, 426, 897

Robertson, I. P., \& Cravens, T. E. 2003, JGR, 108, 6

Richardson, J.D., Wang C., Stone E.C., \& McDonald F.B. 2004, Solar Wind 11, in press

Snowden, S. L., Collier, M. R., \& Kuntz, K. D. 2004, ApJ, 610, 1182

Stone, E. C., \& Cummings, A. C. 2003, in M. Velli et al. (eds.), Solar Wind Ten, AIP Conf. Proc. 679, 47

Stone, E. C., Cummings, A. C., McDonald, F. B., et al 2005, Science, 309, 2017

Vallerga, J., Lallement, R., Lemoine, M., et al, 2004, A\&SA, 426, 855

Wang, C., \& Richardson, J. D. 2003, JGR, 108, 1

Webber, W. R., \& Lockwood, J. A. 2004, JGR, 109, 6107

Witte, M. 2004, A\& $A, 426,835$

Wolff, B., et al, 1999, $A \mathscr{E} A, 346,969$

\section{Solar Wind Modeling and Space Weather}

Stefaan Poedts ${ }^{1}$ and Charles N. Arge $^{2}$

1 (Afdeling Plasma-astrofysica, Katholieke Univ. Leuven, Heverlee Belgium;

stefaan.poedts@wis.kuleuven.be) 
2 (Air Force Research Laboratory, Space Vehicles Directorate, Hanscom AFB, MA USA; nick.arge@hanscom.af.mil)

\subsection{Introduction}

Important new developments occurred in the domain of solar wind modeling from mid2002 to mid-2005. The availability of increasing CPU power and computer memory enables ever more advanced models including new physical and geometrical effects. They often now incorporate observational data as boundary conditions, and the simulations are starting to be realistic enough to tackle specific event studies and to compare the simulation results in detail with the observations.

\subsection{Solar wind models}

Two groups have developed advanced three-dimensional (3-D) coupled magnetohydrodynamic (MHD) models of the inner heliosphere extending from the low solar corona to well beyond Earth orbit: The Center for Space Environment Modeling (CSEM) group at the University of Michigan and the Center for Integrated Space Weather Modeling (CISM) group at Boston University. At the heart of the CSEM coupled model is the Space Weather Modeling Framework (SWMF), a high-performance flexible computational tool that enables coupling state-of-the art models of the solar corona, the solar wind, and solar energetic particles (Gombosi et al. 2004, Toth et al. 2005). The CISM CORHEL model (Luhmann et al. (2004) and references therein) couples the SAIC coronal MAS (Magnetohydrodynamics Around a Sphere) model with Odstrcil's ENLIL solar wind model.

Other groups also developed advanced solar wind models. Lionello et al. (2003) developed a 3-D MHD model of the solar corona and the solar wind. Where the model originally employed a polytropic energy equation, the physics in the algorithm was improved by incorporating thermal conduction along the magnetic field, radiation losses, and heating into the energy equation. Lee et al. (2004) extended the CISM inner heliospheric model CORHEL to 10 AU to investigate how well this solar magnetogram-based, 3-D MHD model describes the solar wind influence on Saturn's magnetosphere. Odstrcil et al. (2004) also developed a coupled numerical wind model where (1) the ambient solar wind is derived from coronal models utilizing photospheric magnetic field observations, and (2) transient disturbances are derived from geometrical and kinematic fitting of coronagraph observations of coronal mass ejections (CMEs). The coronal models used in the Odstrcil algorithm are the SAIC MAS model and the coronal portion of the WangSheeley-Arge (WSA) model (Arge et al. 2004 and references therein). WSA is a simple physics- and empirical-based coronal and solar wind model. It has been significantly improved over the last three years (Arge et al. 2004) and comprehensively validated with observations spanning nearly a full solar cycle (Owens et al. 2005).

Amari et al. (2005) considered a 3-D bipolar magnetic field which is driven into evolution by the slow turbulent diffusion of its normal component on the boundary. By means of a series of numerical simulations in which the magnetic field evolves from different force-free states, they found that the evolution conserves magnetic helicity. Lionello et al. (2005) developed a global MHD model to study the effect of differential rotation on the coronal magnetic field and identified examples of interchange reconnection and other changes of topology of the field.

\subsection{CME simulations superposed on the wind models}

Heliospheric models of CME propagation and evolution provide an important insight into the dynamics of CMEs and are a valuable tool for interpreting interplanetary in - situ 
observations. Moreover, they represent a virtual laboratory for exploring conditions and regions of space that are not conveniently or currently accessible by spacecraft (Riley 2005). Linker et al. (2003) suggested that future computational modeling of interplanetary CMEs is likely to emphasize the need to study coronal initiation and solar wind propagation together. Serious efforts have been undertaken to reach this goal. Roussev et al. (2003a) used observed synoptic magnetograms to drive the CSEM coupled coronasolar wind model and to simulate the solar wind. Roussev et al. (2003b) used this tool to simulate a 3-D, flux-rope model for a CME. This model is based on a loss of equilibrium, i.e., not on an initially unsatisfied force balance as in many earlier (and current) simulations. Manchester et al. (2004a; 2004c) also modeled erupting flux ropes and the resulting CMEs in full 3-D MHD. Sokolov et al. (2004) then included a field-line advection model and this coupled corona-solar wind-solar energetic particle (SEP) model thus includes the energetic particle environment at any point in the inner heliosphere in the simulations. Manchester et al. (2004b) used the SWMF tool to model specific Space Weather events from the Sun to the Earth, including the initiation of the CME and its evolution during its interplanetary propagation. Manchester et al. (2005) focused on the CME shock and sheet structures relevant for particle acceleration while Lugaz et al. (2005) concentrated on the evolution of the density structure of CMEs. Jacobs et al. (2005) also focused on the propagation of fast CME-generated MHD shock waves, and made a first attempt to quantify the effect of the background solar wind model on this evolution by superposing the same simple CME model on three different 2.5-D (axisymmetric) wind models. Chané et al. (2005) then studied the effect of the CME initiation parameters on the CME evolution, in particular the polarity of the initial magnetic flux rope.

Aran et al. (2004) developed a tool for rapid predictions of proton flux and fluence profiles observed during gradual SEP events for the upstream part of the shock. This code, named SOLPENCO (SOLar Particle ENgineering COde), contains a data base with a large set of interplanetary scenarios for SEPs, basically defined by (1) the heliolongitude of the parent solar activity (from E75 to W90), (2) the position of the observer (either at $0.4 \mathrm{AU}$ or $1.0 \mathrm{AU}$ ) and (3) the initial shock velocity (between $750 \mathrm{~km} / \mathrm{s}$ and $1800 \mathrm{~km} / \mathrm{s}$ at 18 solar radii) of the simulated shock. Aran et al. (2005) applied this model to calculate the synthetic flux profiles of the data set after the shock-plus-particle model developed by Lario et al. (1998).

\subsection{Applications for predictions and forecasting}

Many others applied these and other solar wind and CME evolution models to simulate specific events and compare the results to observations or to investigate data sets for testing the models on their predictive capabilities. For example, a first generation, realtime, combined "quiet" and "event-driven" solar wind model has been used to examine more than 600 solar flares since 1997 (during the rise, maximum, and decline of Solar Cycle 23) that were accompanied by metric radio Type II bursts and/or by partial or full halo CMEs, the latter if available in real time. This desktop model is version 2 of the 3-D kinematic Hakamada-Akasofu-Fry model (HAFv.2) that ingests real-time USAF/NOAA optical, radio, and soft X-ray data (via forecaster input) together with source surface (2.5 solar radii) 'quiet-time' solar wind plasma velocity and IMF magnitude and polarity (Fry et al. 2004; 2005 and references therein) as specified by the WSA model that runs in real-time at NOAA/SEC. As an alternative to metric Type II shock speed estimates, CME plane-of-sky speed inputs were also examined and shown to have no advantage (Cho et al. 2003 and Smith et al. 2005). The HAFv.2 model was also used to explore the Voyager 1 and Voyager 2 responses in the outer heliosphere (e.g., see Intriligator et al. 2005). 
Wu et al. (2005b) developed a data-driven, 3-D MHD model to investigate the nonpotentiality of the active-region corona and to determine the criteria for "initiation" of solar eruptive phenomena. The important innovation of this model is its ability to incorporate realistic photospheric dynamics by inclusion of the magnetic measurements (i.e., emerging and submerging magnetic flux) together with the differential rotation, meridional flow, effective diffusion, and cyclonic turbulence effects to drive the model. Using their streamer and flux-rope MHD model, Wu et al. (2004) have numerically examined the Low \& Zhang (2002) suggestion that the two types of CMEs [i.e., constant speed (fast) and accelerated (slow)] are caused by the initial magnetic topology due to the effect of magnetic reconnection processes. The numerical simulation shows, in addition to the magnetic topology, that the solar surface condition also plays an important role on determining the two types of CMEs (see Wu et al. 2005c). To study the CME propagation, Wang et al. (2005) have employed the LASCO and ACE observations of the January 20, 2001 CME-CME interaction event together with their MHD model to investigate the acceleration and deceleration and cannibalization of the CMEs. In addition, Wu et al. (2005a) utilized a 1.5-D adaptive grid MHD model to study the flare-generated shock evolution and geomagnetic storms during the "Halloween" events, October 29 to November 2, 2005. The results show that simulated solar wind velocity temporal profiles successfully matched the observations at L1 (ACE/SWEPAM/SWICS/MAG).

The May 12, 1997 halo CME event was studied extensively using models. Arge et al. (2004; 2005) reproduced (except for the ejecta itself) the observed stream structure for many days before and after the event using the WSA model. Odstrcil et al. $(2004 ; 2005)$ accomplished the same (except that a shorter time interval was considered) using the ENLIL model (driven independently by both MAS and WSA), while also reproducing, within reason, the profile and arrival time of the shock. This was achieved by introducing a simple transient (as described in Section 1.2) within the ambient solution.

\subsection{Conclusion and near future}

In the period mid-2002 to mid-2005, the modeling of the solar wind and of CMEs superposed on this wind has advanced to the stage where individual events can be simulated rather realistically and then compared with observations. The current state-of-the-art 3-D MHD models are beginning to apply magnetogram data as input boundary conditions and are starting to include non-MHD effects, such as solar particle acceleration and kinetic effects.

The numerical 3-D MHD models are capable of predicting large-scale solar wind structures at Earth, provided that appropriate time-dependent boundary conditions are specified near the Sun. These models also provide us with a global picture of the interactions of transient disturbances with solar wind streams. In the applications of the models, the simple events that were first selected are being replaced by even more difficult cases such as the very complicated Halloween storms, a very challenging series of about a dozen $\mathrm{X}$-class solar events.

The CSEM SWMF model (and soon the CISM model) enables a user, at least in principle, to self-consistently model the magnetospheric interaction of solar wind transients by coupling the solar wind model to a comprehensive global magnetosphere-drift, physics-ionosphere model. However, it will be many years before they are routinely used and fully validated.

\section{References}

Arge, C. N., J. G. Luhmann, D. Odstrcil, C. J. Schrijver, \& Y. Li 2004, JASTP, 66, 1295-1309 Arge, C. N., G. de Toma, \& J.G. Luhmann 2005, in K. Sankarasubramanian, M. Penn, \& A. Pevtsov (eds.), Large Scale Structures and their Role in Solar Activity, ASP (in press) 
Amari, T., Luciani, J.F., Aly, J.J., Mikic, Z., \& Linker, J. 2005, ApJ, 595, 1231-1250

Aran, A., Sanahuja, B., \& Lario, D. 2004, Final Report ESA/ESTEC contract 14098/99/NL/MM, April 2004. Available at http://www.am.ub.es/ blai

Aran, A., Sanahuja, B., \& Lario, D. 2005, Ann. Geophys., 23, 1-7

Chané, E, Jacobs, C., Van der Holst, B., Poedts, S., \& Kimpe, D. 2005, A\&\&A, 432, 331-339

Cho, K.-S., Moon, Y.-J., Dryer, M., Fry, C.D., Park, Y.-D., \& Kim, K.-S. 2003, JGR, 108, No. A12, 1445, doi: 1029.2003JA010029

Fry, C.D. et al. 2004, IEEE Trans. on Plasma Sci., 32, 1489-1497

Fry, C.D. et al. 2005, AIAA J., 43(5), 987-993

Gombosi, T.I. et al. 2004, Comp. in Sci. \& Eng., 6, No 2, 14-35

Intriligator, D.S., Sun, W., Dryer, M., Fry, C.D., Deehr, C.S., \& Intriligator, J. 2005, JGR, 110, A09S10, doi:10.1029/2005JA011011

Jacobs, C., Poedts, S., Van der Holst, B., \& Chané, E. 2005, A\&A A, 430, 1099-1107

Lario, D., Sanahuja, B., \& Heras, A.M. 1998, ApJ, 509, 415-434

Lee, C.O. et al. 2004, EOS Trans. AGU 84(46), Fall Meet. Suppl., Abstract P51A-A1418

Linker, J.A., Mikić, Z., Riley, P., Lionello, R., \& Odstrcil, D. 2003, in M. Velli et al. (eds.), Solar Wind Ten, AIP Conf. Proc. 679, 703-710

Lionello, R., Linker, J.A., \& Mikić, Z., 2003: in M. Velli et al. (eds.), Solar Wind Ten, AIP Conf. Proc. 679, 222-225

Lionello, R., Riley, P., Linker, J.A., \& Mikić, Z. 2005, ApJ, 625, 463L

Lugaz, N., Manchester W.B., \& Gombosi T.I. 2005, ApJ, 627, 1019-1030

Luhmann, J. G. S. C. Solomon, J. A. Linker, J. G. Lyon, Z. Mikic, D. Odstrcil, W. Wang, \& M. Wiltberger 2004, JASTP, 66, 1243

Low, B.C. \& Zhang, M. 2002, ApJ, 564, L53-L56

Manchester, W.B., Gombosi, T.I., De Zeeuw, D.L., \& Fan, Y. 2004a, ApJ, 610, 588-596

Manchester, W.B., Gombosi, T.I., Ridley, A.J., Roussev, I.I., De Zeeuw, D.L., Sokolov, I.V., Powell, K.G., \& Toth, G. 2004b, JGR, 109, A02107, doi:10.1029/2003JA010150

Manchester, W.B., Gombosi, T.I., Roussev, I.I., De Zeeuw, Sokolov, I.V., Powell, K.G., Toth, G., \& Opher, M. 2004c, JGR, 109, A01102, doi:10.1029/2002JA009672

Manchester, W.B. et al. 2005, ApJ, 622, 1225-1239

Odstrcil, D., Riley, P., \& Zhao, X.P. 2004, JGR, 109, A02116, doi:10.1029/2003JA010135

Odstrcil, D., Pizzo, V.J., \& Arge, C.N. 2005, JGR, 110, A02106, doi:10.1029/2004JA010745

Owens, M. J., Arge, C. N., Spence, H. E., \& Pembroke, A. 2005, JGR, (in press)

Riley, P. 2005, in K. Dere, J. X. Wang, \& Y. Yan (eds.), Coronal and Stellar Mass Ejections, Proc. of IAU, vol. 2004;IAUS226, CUP, 389-402

Roussev, I.I. et al. 2003a, ApJ, 595, L57-L61

Roussev, I.I., Forbes, T.G., Gombosi, T.I., Sokolov, I.V., De Zeeuw, D.L., \& Birn, J. 2003b, ApJ, 588, L45-L48

Smith, Z., Dryer, M., \& Fry, C.D. 2005, Space Weather, 3, S07002, doi:10.1029/2004SW000136

Sokolov, I.V., Roussev, I.I., Gombosi, T.I., Lee, M.A., Kota, J., Forbes, T.G., Manchester, W.B., \& Sakai, J.I. 2004, ApJ, 616, L171-L174

Toth, G. et al. 2005, in Lui, A.T.Y., Kamide, Y., \& Consolini, G. (eds.), Multiscale Coupling of Sun-Earth Processes, 383-397, Elsevier

Wang, A. H., S.T. Wu, \& N. Gopalswamy 2005, in D. Gallagher, J. Horwitz, J. Perez, R. Preece \& J. Quenby (eds.), Particle Acceleration in Astrophysical Plasmas, GM 156, 185-195, AGU

Wu, C.C., S.T. Wu, \& M. Dryer 2004, SPh, 223, 259-282

Wu, C.C. et al. 2005a, JGR, 110, doi:10.1029/2005JA011011

Wu, S.T., A.H. Wang, \& D.A. Falconer 2005b, in K. Dere, J. X. Wang, \& Y. Yan (eds.), Coronal and Stellar Mass Ejections, Proc. of IAU, vol. 2004;IAUS226, CUP, 291-301

Wu, S.T., T.X. Zhang, E. Tandberg-Hanssen, Y. Liu, Xueshang Feng, \& A. Tan 2005c, SPh, $225,157-175$ 